\title{
Efecto de la temperatura de calcinación en catalizadores FeMo/Zn- Hidroxiapatitas sobre la reacción de hidrodesulfuración de tiofeno
}

\author{
Effect of calcination temperature of FeMo/Zn-Hydroxyapatites catalysts \\ on the thiophene hydrodesulfuration reaction
}

\author{
Edith Figueredo ${ }^{1,2 *} \quad$ Carlos F. Linares $^{1} \quad$ Freddy Ocanto $^{1}$ \\ Recibido 01 de agosto de 2017, aceptado 25 de junio de 2018 \\ Received: August 01, 2017 Accepted: June 25, 2018
}

\begin{abstract}
RESUMEN
Se sintetizaron soportes de Zn-hidroxiapatitas con contenido variable de $\mathrm{Zn}$ (0-15\% mol Zn); luego fueron impregnados con Mo, como fase activa, y Fe, como promotor. Posteriormente, los sólidos se dividieron en dos porciones: una parte fue calcinada a $400{ }^{\circ} \mathrm{C}$ y la otra a $800{ }^{\circ} \mathrm{C}$ por $4 \mathrm{~h}$. Todas estas muestras fueron caracterizadas mediante diversas técnicas fisicoquímicas tales como: difracción de rayos X (DRX), espectroscopía de infrarrojo con transformada de Fourier, microscopía electrónica de barrido, reducción a temperatura programada y medidas de área específica por el método BET. Las fases identificadas por DRX fueron: $\mathrm{Fe}_{2} \mathrm{Mo}_{3,9} \mathrm{O}_{14,7} 10.5 \mathrm{H}_{2} \mathrm{O}, \mathrm{Fe}_{2}\left(\mathrm{MoO}_{4}\right)_{3}, \mathrm{Ca}_{19} \mathrm{Zn}_{2}\left(\mathrm{PO}_{4}\right)_{14}$ y $\mathrm{CaMoO}_{4}$ (scheelita), para las dos temperaturas de calcinación. Finalmente, los precursores fueron empleados en la reacción de hidrodesulfuración de tiofeno, obteniéndose bajos valores de actividad catalítica para los sólidos calcinados a $800{ }^{\circ} \mathrm{C}$, posiblemente debido a las menores superficies específicas y a la poca presencia del molibdeno octaédrico en dichos sólidos.
\end{abstract}

Palabras clave: Hidrotratamiento, tiofeno, hidroxiapatita, temperatura de calcinación.

\begin{abstract}
Zn-hydroxiapatites supports were synthesized with a variable Zn content $(0-15 \% \mathrm{~mol} Z n)$. Then, they were impregnated with Mo, as phase active, and $\mathrm{Fe}$, as the promoter. Solids were subsequently divided into two parts: one portion was calcined at $400^{\circ} \mathrm{C}$ and another one at $800^{\circ} \mathrm{C}$ for $4 \mathrm{~h}$. All samples were characterized using various physico-chemical techniques such as: X-ray diffraction, (XRD), Fourier transformed infrared spectroscopy, scanning electron microscopy, temperature programmed reduction and specific area measurements by BET method. XRD determined identified phases such as: $\mathrm{Fe}_{2} \mathrm{Mo}_{3.9} \mathrm{O}_{14.7} 10.5 \mathrm{H}_{2} \mathrm{O}$, $\mathrm{Fe}_{2}\left(\mathrm{MoO}_{4}\right)_{3}, \mathrm{Ca}_{19} \mathrm{Zn}_{2}\left(\mathrm{PO}_{4}\right)_{14}$ and $\mathrm{CaMoO}_{4}$ (scheelite) for both calcination temperatures for both calcination temperatures. Finally, catalytic precursors were used in the hydrodesulfuration reaction of thiophene, obtaining low catalytic activity values for solids calcined at $800{ }^{\circ} \mathrm{C}$; possibly due to the low surface areas and the low presence of octahedral molybdenum for these solids.
\end{abstract}

Keywords: Hydrotreating, thiophene, hydroxyapatites, calcination temperature.

\footnotetext{
1 Unidad de Síntesis de Materiales y Metales de Transición (SIMMET). Departamento de Química. Facultad de Ciencias y Tecnología. Universidad de Carabobo, Valencia. Edo. Carabobo, Venezuela. E-mail: clinares@uc.edu.ve; ocantow@gmail.com

2 Laboratorio de Calidad Ambiental. Escuela de Civil, Facultad de Ingeniería. Universidad de Carabobo, Valencia. Edo. Carabobo, Venezuela. E-mail: emfigueredo@gmail.com

* Autor de correspondencia
} 


\section{INTRODUCCIÓN}

El hidrotratamiento (HDT), constituye uno de los procesos catalíticos más importantes practicados en las refinerías modernas. Su objetivo principal es remover compuestos orgánicos con heteroátomos como azufre, nitrógeno, oxígeno o metales, así como los aromáticos presentes en el crudo o en fracciones derivadas del mismo. Estas reacciones suelen llevarse a cabo en presencia de hidrógeno, altas temperaturas y catalizadores Co-Mo o Ni-Mo soportados en alúmina.

Con la finalidad de proteger el ambiente, muchos países han adoptado normas y leyes que regulan la cantidad de compuestos sulfurados, nitrogenados, oxigenados y aromáticos permitidos en las fracciones del petróleo, así como sus consecuentes emisiones a partir de la combustión [1]. Actualmente, los trabajos en hidrotratamiento están orientados a modificar el soporte utilizado tradicionalmente $\left(\gamma-\mathrm{Al}_{2} \mathrm{O}_{3}\right)$ o sustituirlo por otros [2], debido a que son poco efectivos para las cargas de petróleo procesadas, lo que conlleva a la necesidad de mejorar y proponer nuevos catalizadores que permitan aportar soluciones a los problemas generados al hacer uso de los catalizadores convencionales. Así, nuevos materiales mesoporosos, carbones, hidrotalcitas e hidroxiapatitas son prometedores de resultados interesantes [3].

En este sentido, una alternativa la constituye el uso de hidroxiapatita fosfocálcica: $\mathrm{Ca}_{10}\left(\mathrm{PO}_{4}\right)_{6}(\mathrm{OH})_{2}$, perteneciente a la familia cristalográfica de las apatitas, compuestas por isomorfos que poseen una misma estructura hexagonal.

Esta investigación plantea el uso de hidroxiapatita como soporte catalítico, promovidas con Fe y Mo como fases activas. Los resultados, usando hierro como promotor, son ambiguos, algunos autores indican su poco efecto como promotor, mientras que otros afirman un efecto positivo, en algunas reacciones como la hidrodesmetalización de crudos pesados [4]. Asimismo, se ha observado sinergia en reacciones de hidrotratamiento cuando el sulfuro de niobio es promovido con hierro [5].

El propósito de esta investigación fue sintetizar hidroxiapatitas de contenido $\mathrm{Ca}-\mathrm{Zn}$ variable y utilizarlas como un nuevo soporte de catalizadores de reacciones de hidrodesulfuración. Además, se estudia el efecto de la temperatura de calcinación $\left(400\right.$ y $800{ }^{\circ} \mathrm{C}$ ) con el fin de comparar este efecto en las propiedades texturales de dichos precursores catalíticos y su influencia en la actividad catalítica, ya que estudios previos han demostrado que la temperatura de calcinación influye notablemente en las propiedades físicas y químicas de los potenciales catalizadores utilizados en hidrotratamiento [6-7].

\section{PARTE EXPERIMENTAL}

Las Zn-hidroxiapatitas fueron sintetizadas por el método de co-precipitación previamente reportado por Miyaji, [8]. En consecuencia, una solución acuosa (solución A) fue preparada disolviendo $\left(\mathrm{NH}_{4}\right)_{2} \mathrm{HPO}_{4}$ en agua destilada con una concentración en $[\mathrm{P}]=0,06$ mol.* $\mathrm{L}^{-1}$. Una solución $\mathrm{B}$ fue también preparada mezclando $\mathrm{Ca}\left(\mathrm{CH}_{3} \mathrm{COO}\right)_{2} \mathrm{H}_{2} \mathrm{O}$ y $\mathrm{Zn}\left(\mathrm{CH}_{3} \mathrm{COO}\right)_{2}$ $2 \mathrm{H}_{2} \mathrm{O}$ en agua destilada con una concentración $[\mathrm{Zn}+\mathrm{Ca}]=0,1 \mathrm{~mol} * \mathrm{~L}^{-1}$. La fracción para cada composición, de 0 a $15 \%$ mol, fue representada como $([\mathrm{Zn}] /[\mathrm{Zn}+\mathrm{Ca}]) * 100(\% \mathrm{~mol})$. Luego, la solución $B$ fue añadida lentamente sobre la solución A bajo agitación vigorosa a $90^{\circ} \mathrm{C}$ por $5 \mathrm{~h}$. El pH fue ajustado a 8 usando una solución diluida de hidróxido de amonio. Después, la suspensión formada fue filtrada y lavada con abundante agua destilada hasta alcanzar un valor de $\mathrm{pH}$ cercano a 7. Los productos, polvos de tonalidad blanquecina, fueron secados a $80^{\circ} \mathrm{C}$ por 24h. Cada sólido seco fue impregnado por el método de humedad incipiente con Mo (heptamolibdato de amonio $\left[\left(\mathrm{NH}_{4}\right)_{6} \mathrm{Mo}_{7} \mathrm{O}_{24} 4 \mathrm{H}_{2} \mathrm{O}\right]$, Riedel de Haën al 99\%) como fase activa, y Fe como promotor (sal de Mohr $\left[\left(\mathrm{NH}_{4}\right)_{2} \mathrm{SO}_{4} \mathrm{FeSO}_{4} 6 \mathrm{H}_{2} \mathrm{O}\right]$, Fisher Scientific al 99\%). El sólido fue impregnado con Mo composición nominal de $15 \%$ como $\mathrm{MoO}_{3}$. Luego, el sólido fue secado a $80^{\circ} \mathrm{C}$ por $24 \mathrm{~h}$ y co-impregnado con $\mathrm{Fe}$ en proporción atómica $3 \mathrm{Mo}$ :Fe. Los sólidos fueron secados a temperatura ambiente y divididos en dos porciones: una porción fue calcinada a $400{ }^{\circ} \mathrm{C}$ y la otra porción a $800{ }^{\circ} \mathrm{C}$ por $4 \mathrm{~h}$. Los catalizadores fueron identificados como FeMo/Hap (n\%Zn), donde Hap es la fase hidroxiapatita y $\mathrm{n}$ es el porcentaje molar de Zn entre 0 y $15 \%$.

Las Zn-hidroxiapatitas sintetizadas se caracterizaron mediante técnicas fisicoquímicas tales como: difracción de rayos X de polvos (DRX), medidas de área específica por el método BET y espectroscopía de infrarrojo con transformada de Fourier (FT-IR). 
Por su parte, los precursores catalíticos, $\mathrm{FeMo} /$ Zn-hidroxiapatitas fueron caracterizados por las técnicas anteriormente mencionadas, así como por microscopía electrónica de barrido (MEB) y reducción a temperatura programada (TPR).

Los estudios de DRX fueron hechos en un difractómetro Siemens (D500S) con radiación CuK $\alpha$ $(1,54060 \AA$ Å) para la detección de la fase cristalina entre 5 y $80^{\circ}(2 \theta)$. La presencia de los grupos funcionales de los sólidos fue evaluada por FT-IR; los espectros fueron registrados en un espectrómetro Shimadzu 8400 S, en el intervalo de 4000-500 $\mathrm{cm}^{-1}$. Las muestras fueron preparadas mezclando los sólidos con $\mathrm{KBr}$ para formar una pastilla fina. Las medidas de área específica por el método BET, fueron llevadas a cabo en un Quantachrome instrument usando $\mathrm{N}_{2}$ como adsorbato. Los análisis TPR se realizaron en un Chemisorb Analizer $2900 \mathrm{de}$ Micromeritics. Las muestras fueron calentadas desde temperatura ambiente hasta $950^{\circ} \mathrm{C}$, a una velocidad de calentamiento de $5^{\circ} \mathrm{C}^{*} \mathrm{~min}^{-1}$, bajo una corriente gaseosa de $\mathrm{H}_{2} / \mathrm{Ar}(10 / 90 \mathrm{v} / \mathrm{v})$, usando un flujo de $50 \mathrm{ml}^{*} \mathrm{~min}^{-1}$. Los análisis de MEB se realizaron en un microscopio Philips XL 30 operando a $20 \mathrm{keV}$.

La reacción de hidrodesulfuración (HDS) de tiofeno se efectuó en un reactor de flujo continuo a presión atmosférica. Todos los catalizadores fueron previamente sulfurados con una solución de $\mathrm{CS}_{2}(10$ $\mathrm{v} / \mathrm{v} \%, 10 \mathrm{~mL}^{*} \mathrm{~h}^{-1}$ )/heptano bajo una corriente de $\mathrm{H}_{2}$ $\left(100 \mathrm{~mL}^{*} \mathrm{~min}^{-1}\right)$. La temperatura se incrementó hasta $400^{\circ} \mathrm{C}$, a una velocidad de $0,0833^{\circ} \mathrm{C}^{*} \mathrm{~s}^{-1}$, manteniendo estas condiciones por $2 \mathrm{~h}$. Luego, la reacción tomó lugar usando $200 \mathrm{mg}$ de catalizador empleando una alimentación líquida $\left(2,710^{-4} \mathrm{~mL}^{*} \mathrm{~s}^{-1}\right)$ compuesta por $10 \mathrm{p} / \mathrm{p} \%$ de tiofeno en heptano y una corriente de $\mathrm{H}_{2}\left(0,25 \mathrm{~mL} \cdot \mathrm{s}^{-1}\right)$ a $280{ }^{\circ} \mathrm{C}$.

El sistema se cubrió con una manta de calentamiento $\left(150^{\circ} \mathrm{C}\right)$ con el fin de evitar la condensación de los productos de reacción. Los productos de reacción se inyectaron en forma gaseosa en un cromatógrafo de gases Varian 3800 (AutoSystem XL) equipado con detector FID usando una columna capilar (5\% bifenilo y $95 \%$ de dimetilsilano) de $30 \mathrm{~m}$ de longitud y un diámetro interno de $0,25 \mathrm{~mm}$.

El catalizador comercial fue preparado utilizando como soporte $\gamma$-alúmina, el proceso de impregnación, calcinación, activación y prueba catalítica se llevaron a cabo empleando el mismo procedimiento descrito anteriormente para los soportes de la hidroxiapatita. Estos sólidos fueron caracterizados por reducción a temperatura programada (TPR) y medidas de área específica por el método de BET.

\section{RESULTADOS Y DISCUSIÓN}

La Figura 1 señala los difractogramas de las muestras de las $\mathrm{Zn}$-hidroxiapatitas sintetizadas (0-15\% Zn mol).

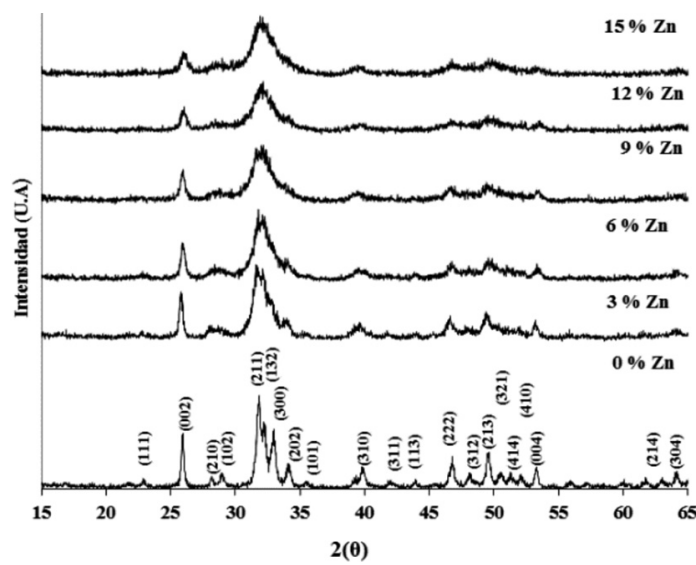

Figura 1. Difractogramas de las muestras sintetizadas para diferentes composiciones molares de Zn. Leyenda: 0\% Zn Hap (JCDPS 09-0432).

Estos difractogramas muestran picos intensos correspondiente a los planos: (211), (002), (132), (300), (222) y (213) los cuales son representativos de las estructuras hidroxiapatitas (JCDPS 09-0432) [4]. No se identificaron otras fases.

Adicionalmente, a medida que el contenido de $\mathrm{Zn}$ aumenta, la intensidad de los picos disminuye y comienzan a hacerse más anchos. Esto podría estar asociado al tamaño de los cristalitos los cuales son más pequeños a medida que el contenido de $\mathrm{Zn}$ incrementa [8].

Por otro lado, la Figura 2 muestra los difractogramas correspondientes a las muestras seleccionadas de los precursores catalíticos, FeMo/Hap $(0 \% \mathrm{Zn})$, $\mathrm{FeMo} / \mathrm{Hap}(6 \% \mathrm{Zn})$ y FeMo/Hap $(15 \% \mathrm{Zn})$ calcinadas a $400{ }^{\circ} \mathrm{C}$. Para la muestra $\mathrm{FeMo} / \mathrm{Hap}(0 \% \mathrm{Zn})$, se identificaron las siguientes fases: hidroxiapatita, 


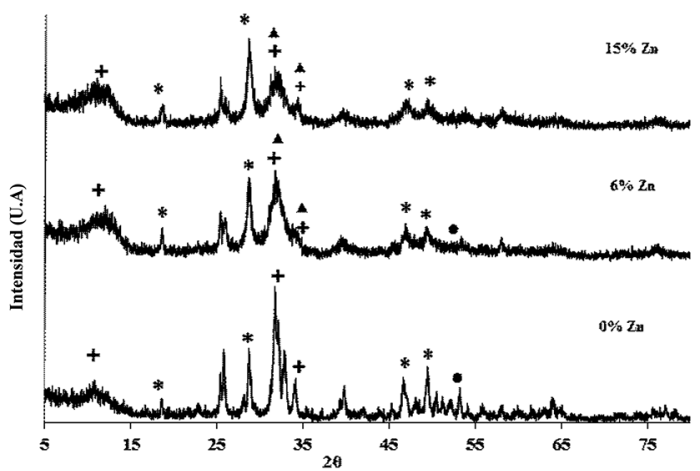

Figura 2. Difractogramas de las muestras FeMo/ Zn-hidroxiapatitas calcinadas a $400^{\circ} \mathrm{C}$. Leyenda: * Fase scheelita $\mathrm{CaMoO}_{4}$, + Fase ferrimolibdito $\mathrm{Fe}_{2} \mathrm{Mo}_{3.9} \mathrm{O}_{14.7} 10.5 \mathrm{H}_{2} \mathrm{O}$, -Fase molibdato férrico $\mathrm{Fe}_{2}\left(\mathrm{MoO}_{4}\right)_{3}$, $\Delta$ Fase fosfato de calcio y cinc $\mathrm{Ca}_{19} \mathrm{Zn}_{2}$ $\left(\mathrm{PO}_{4}\right)_{14}$.

scheelita $\mathrm{CaMoO}_{4}$ (JPDCS 77-2238), ferrimolibdito $\mathrm{Fe}_{2} \mathrm{Mo}_{3.9} \mathrm{O}_{14.7} 10.5 \mathrm{H}_{2} \mathrm{O}$ (JPDCS 15-0289) y molibdato férrico $\mathrm{Fe}_{2}\left(\mathrm{Mo}_{4} \mathrm{O}_{4}\right)_{3}$ (JPDCS 20-0526).

La scheelita se forma por la reacción a alta temperatura entre el $\mathrm{Ca}$, que migra fuera de la estructura de la hidroxiapatita, y el heptamolibdato de amonio impregnado. Esta fase fue encontrada en todos los sólidos calcinados a 400 y $800^{\circ} \mathrm{C}$. La fase ferrimolibdito, así como la fase molibdato férrico, posiblemente se formaron por la reacción a altas temperaturas entre el heptamolibdato de amonio y la sal de Mohr utilizadas en el proceso de impregnación de los sólidos. Para las muestras con contenido de cinc se identificó, adicionalmente, la fase fosfato de calcio y cinc $\mathrm{Ca}_{19} \mathrm{Zn}_{2}\left(\mathrm{PO}_{4}\right)_{14}$ (JPDCS 48-1196) [6]. Esta última fase se formó durante el proceso de calcinación de la Zn-hidroxiapatita.

La Figura 3 muestra las diferentes fases encontradas después de la calcinación del sólido a $800{ }^{\circ} \mathrm{C}$. Las fases identificadas para las muestras $\mathrm{FeMo} /$ Hap $(0 \% \mathrm{Zn}), \mathrm{FeMo} / \mathrm{Hap}(6 \% \mathrm{Zn})$ y $\mathrm{FeMo} / \mathrm{Hap}$ $(15 \% \mathrm{Zn})$ calcinadas a $800{ }^{\circ} \mathrm{C}$, fueron las mismas identificadas para el sólido calcinado a $400{ }^{\circ} \mathrm{C}$. También ocurre un solapamiento de las señales de fosfato de calcio y cinc $\mathrm{Ca}_{19} \mathrm{Zn}_{2}\left(\mathrm{PO}_{4}\right)_{14}$ con las señales del molibdato férrico, correspondientes a ferrimolibdito. La presencia de calcio y fosfato de cinc se produce por la deshidroxilación de la Zn-hidroxiapatita [8-9].

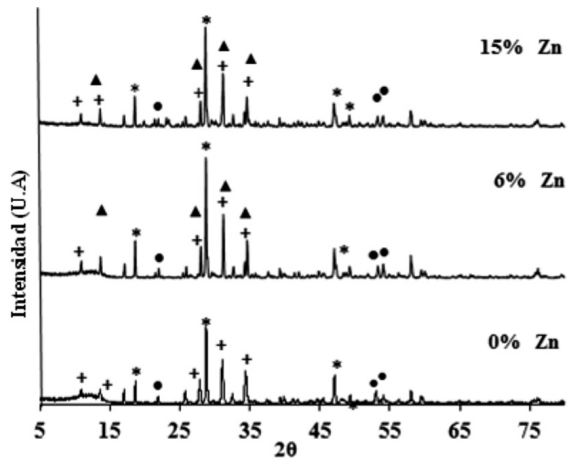

Figura 3. Difractogramas de las muestras $\mathrm{FeMo} /$ Zn-hidroxiapatitas calcinadas a $800^{\circ} \mathrm{C}$. Leyenda: * Fase scheelita $\mathrm{CaMoO}_{4}$, + Fase ferrimolibdito $\mathrm{Fe}_{2} \mathrm{Mo}_{3.9} \mathrm{O}_{14.7} 10.5 \mathrm{H}_{2} \mathrm{O}$, - Fase molibdato férrico $\mathrm{Fe}_{2}\left(\mathrm{MoO}_{4}\right)_{3}$, $\Delta$ Fase fosfato de calcio y cinc $\mathrm{Ca}_{19} \mathrm{Zn}_{2}\left(\mathrm{PO}_{4}\right)_{14}$.

Al comparar los difractogramas de los últimos precursores catalíticos analizados, se observa una mejor definición de las señales de respuesta al aumentar la temperatura de calcinación. Este hecho está directamente relacionado con el tamaño de los cristales. Los sólidos calcinados a $400^{\circ} \mathrm{C}$ presentan cristales 10 veces más pequeños que los sólidos calcinados a $800^{\circ} \mathrm{C}$, tal como lo refleja la Tabla 1 . Este resultado está de acuerdo a lo reportado por Spadavecchia y González [10], quienes encontraron que en hidroxiapatitas modificadas con $\mathrm{Ni}^{2+}, \mathrm{Mo}^{6+} \mathrm{y}$ $\mathrm{Co}^{2+}$ calcinadas a $800{ }^{\circ} \mathrm{C}$, se incrementa el tamaño del cristal al aumentar la temperatura por efecto de la deshidroxilación parcial de la hidroxiapatita durante la calcinación.

En cuanto al tamaño del cristal, en la Tabla 1 se observa, que el soporte tipo Hap $0 \% \mathrm{Zn}$, presentó el mayor tamaño de cristal en comparación con los demás soportes. Los valores del tamaño de los cristales fueron determinados a partir de la ecuación de Scherrer usando la reflexión del plano (310) $(2 \theta=$ 39,96, PDF-84-1998, beta corregido con nitrato de plata estándar).

A medida que aumenta el contenido de cinc en el sólido, se observa una disminución del tamaño del cristal para los soportes 6, 9 y $12 \% \mathrm{Zn}$. Este resultado es concordante con lo observado en los difractogramas de estas muestras (Figura 1), en donde se aprecia que la intensidad de los picos disminuye y comienzan a 
Tabla 1. Tamaño de los cristales de los soportes sintetizados y de los precursores calcinados a 400 y $800{ }^{\circ} \mathrm{C}$ usando la ecuación de Scherrer.

\begin{tabular}{|c|c|c|c|}
\hline \multirow[b]{2}{*}{ Soportes } & \multicolumn{3}{|c|}{ Tamaño de los cristales (nm) } \\
\hline & $\begin{array}{l}\text { Hidroxiapatita } \\
(\text { Hap) sintetizada } \\
( \pm \mathbf{0 , 1})\end{array}$ & $\begin{array}{c}\mathrm{FeMo/Hap} \\
\text { calcinadas a } 400{ }^{\circ} \mathrm{C} \\
( \pm \mathbf{0 , 1})\end{array}$ & $\begin{array}{c}\mathrm{FeMo/Hap} \\
\text { calcinadas a } 800{ }^{\circ} \mathrm{C} \\
( \pm 1)\end{array}$ \\
\hline Hap 0\% Zn & 19,4 & 12,0 & 65 \\
\hline Hар 6\% Zn & 9,2 & 7,8 & 78 \\
\hline Hap 9\% Zn & 9,0 & 5,3 & 78 \\
\hline Нар 12\% Zn & 8,4 & 4,9 & 72 \\
\hline Hap 15\% Zn & 10,2 & 5,0 & 74 \\
\hline
\end{tabular}

hacerse más anchos, cuando la muestra contiene cinc. Según Miyaji y col [8], el tamaño de los cristalitos es más pequeño a medida que el contenido de $\mathrm{Zn}$ incrementa, afectando su cristalinidad aparente.

La Figura 4 muestra el espectro FT-IR de las muestras FeMo/Zn-hidroxiapatitas calcinadas a $400{ }^{\circ} \mathrm{C}$. Se observaron las bandas de $\mathrm{PO}_{4}{ }^{3-}$ alrededor de 1101, 1032, y $564 \mathrm{~cm}^{-1}$. Estas bandas corresponden a los modos de vibración $\mu_{3}$ [11]. Además, se observa la banda a $805 \mathrm{~cm}^{-1}$ asociada a la fase scheelita $\mathrm{CaMoO}_{4}$ [10]. Esta fase fue también determinada mediante DRX. La intensidad de esta banda se incrementa ligeramente a medida que

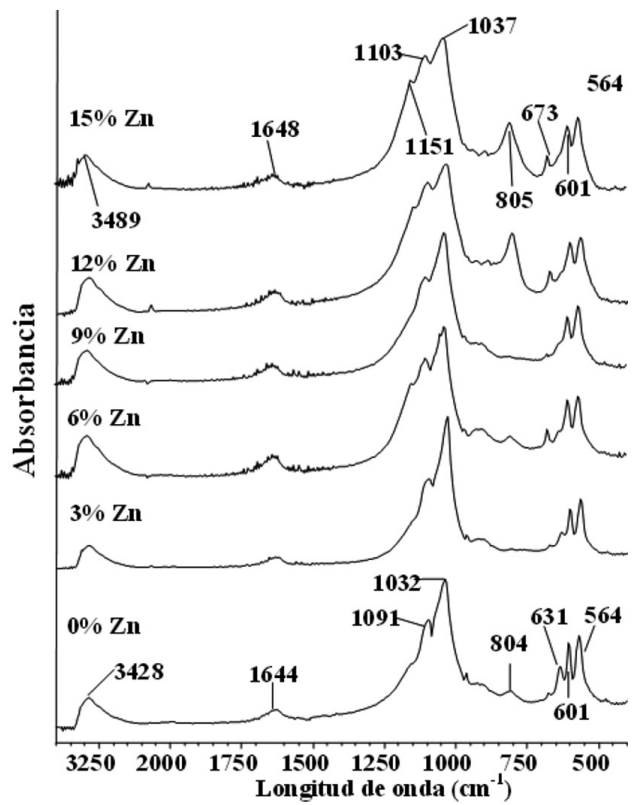

Figura 4. Espectros FTIR de las muestras FeMo/ Zn-hidroxiapatitas calcinadas a $400^{\circ} \mathrm{C}$. aumenta el contenido de $\mathrm{Zn}$. Esta fase de $\mathrm{CaMoO}_{4}$ pudiese disminuir el contenido de especies de Mo octaédrico útiles para la reacción de HDS debido a su difícil reducción, ya que el Mo se encuentra en coordinación tetraédrica.

También se observan bandas alrededor de 632, 3420 y $1600 \mathrm{~cm}^{-1}$ para todas las muestras sintetizadas y calcinadas a $400^{\circ} \mathrm{C}$, las cuales se corresponden con las moléculas de agua adsorbida o los grupos $\mathrm{OH}^{-}$de la hidroxiapatita.

La banda a $1091 \mathrm{~cm}^{-1}$ puede ser atribuida a los aniones $\mathrm{HPO}_{4}{ }^{2-}$. Generalmente los aniones $\mathrm{HPO}_{4}{ }^{2-}$ pueden generar una banda menos intensa a 875 $\mathrm{cm}^{-1}$ la cual puede estar solapada con la banda a $916 \mathrm{~cm}^{-1}$ atribuida a los aniones fosfatos $[10,12]$.

Los espectros de infrarrojo correspondientes a los precursores catalíticos calcinados a $800^{\circ} \mathrm{C}$ se muestran en la Figura 5. Las bandas de fosfatos de estos sólidos, aparecen ligeramente desplazadas en comparación con las muestras calcinadas a $400{ }^{\circ} \mathrm{C}$ producto de la transformación de la hidroxiapatita a especies de fosfato [8]. La banda a $813 \mathrm{~cm}^{-1}$ corresponde a la fase scheelita [13]. Las bandas a 631, 1644 y $3420 \mathrm{~cm}^{-1}$ observadas en las muestras FeMo/Zn-hidroxiapatitas calcinadas a $400^{\circ} \mathrm{C}$, y adjudicadas a las vibraciones $\mathrm{OH}^{-}$no se encontraron en los espectros de infrarrojo de las muestras calcinadas a $800{ }^{\circ} \mathrm{C}$ producto de la deshidratación de las mismas. Las bandas a 1140 y $1036 \mathrm{~cm}^{-1}$ corresponden a las bandas de vibración $\mu_{3}$ de $\mathrm{PO}_{4}{ }^{3-}$, y la banda $552 \mathrm{~cm}^{-1}$ corresponden a la vibración $\mu_{4}$ de fosfatos [11].

Las bandas a 1091 y $875 \mathrm{~cm}^{-1}$ corresponden a las bandas de vibración $\mu_{3}$ del anión $\mathrm{HPO}_{4}{ }^{2-}$. [10, 14] 


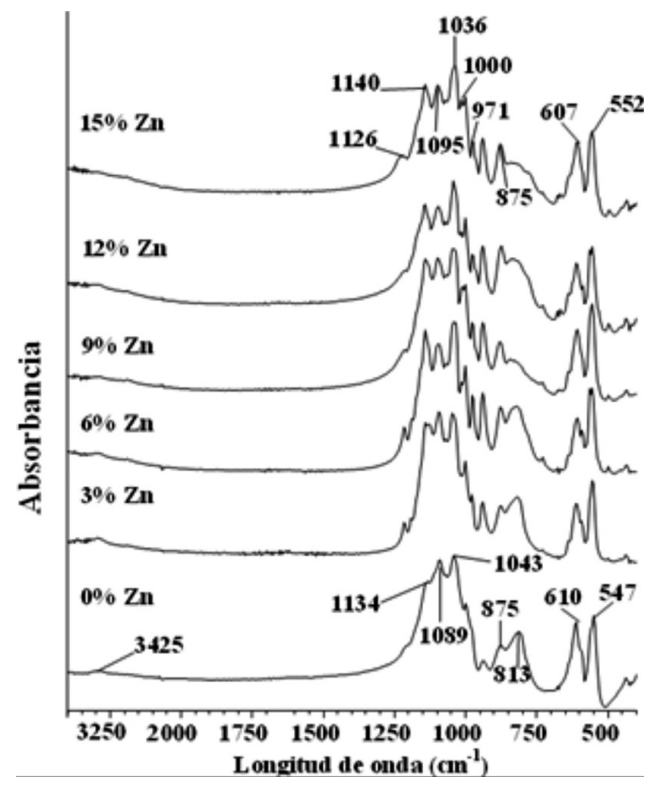

Figura 5. Espectros FTIR de las muestras FeMo/ Zn-hidroxiapatitas calcinadas a $800^{\circ} \mathrm{C}$.

Asimismo, los precursores catalíticos fueron caracterizados por medidas de área específica por el método BET, (Tabla 2). En esta Tabla se resalta la gran diferencia de valores de superficie específica obtenidos para los sólidos bajo estudio y por supuesto la influencia de la temperatura. Esta diferencia de área específica podría deberse al tamaño del cristal al incrementarse la temperatura e impregnarse con los metales de interés. Así, los sólidos calcinados a $400^{\circ} \mathrm{C}$, presentan superficies específicas mayores, en comparación a las obtenidas por los sólidos calcinados a mayor temperatura, por presentar tamaño de cristal más pequeño; estos resultados concuerdan con los reportados en la bibliografía [7-8].

En la Figura 6 se observa la imagen MEB de la muestra FeMo/Zn-hidroxiapatita $(0 \% \mathrm{Zn})$ calcinada a $400^{\circ} \mathrm{C}$, cuyas partículas muestran, en general, una morfología irregular. Sin embargo, ahondado en los detalles, se pueden observar la existencia de partículas alargadas y extremos redondeados semejantes a bastones con un promedio de longitud entre 4 y $20 \mu \mathrm{m}$ y un ancho entre 2 y $5 \mu \mathrm{m}$ aproximadamente asignada a la fase hidroxiapatita [11], y una segunda morfología de aglomerados cúbicos de partículas, con longitudes promedio de 4 y $40 \mu$ m y 4 y $80 \mu$ m aproximadamente correspondientes a las fases scheelita $\mathrm{CaMoO}_{4}$, molibdato férrico, ferrimolibdito y fosfato de calcio.
Tabla 2. Área específica por el método BET, obtenida para las muestras $\mathrm{FeMo} / \mathrm{Zn}$-hidroxiapatitas calcinadas a 400 y $800{ }^{\circ} \mathrm{C}$.

\begin{tabular}{|c|c|c|}
\hline \multirow{2}{*}{ Sólidos } & \multicolumn{2}{|c|}{$\begin{array}{c}\text { Área específica } \\
\text { por el método BET } \\
\left( \pm 1 \mathbf{~ m}^{2} / \mathbf{g}\right)\end{array}$} \\
\hline & $\begin{array}{c}\text { FeMo/Hap } \\
\text { calcinadas a } \\
400^{\circ} \mathrm{C}\end{array}$ & $\begin{array}{c}\text { FeMo/Hap } \\
\text { calcinadas a } \\
800^{\circ} \mathrm{C}\end{array}$ \\
\hline Hap 0\% Zn & 75 & 5 \\
\hline Hap 3\% Zn & 74 & 5 \\
\hline Hap 6\% Zn & 106 & 2 \\
\hline Hap $9 \% \mathrm{Zn}$ & 95 & 2 \\
\hline Hap $12 \% \mathrm{Zn}$ & 85 & 6 \\
\hline Hap $15 \% \mathrm{Zn}$ & 99 & 4 \\
\hline
\end{tabular}

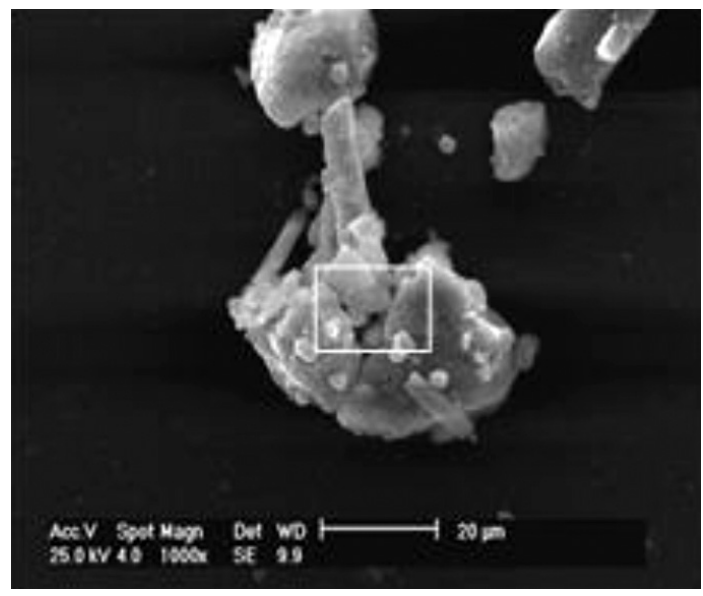

Figura 6. Fotomicrografía MEB de $\mathrm{FeMo} / \mathrm{Zn}$ hidroxiapatita $(0 \% \mathrm{Zn})$ calcinada a $400{ }^{\circ} \mathrm{C}$.

La Figura 7 corresponde a el espectro EDS de la muestra FeMo/ Zn-hidroxiapatita ( $0 \% \mathrm{Zn}$ ) calcinada a $400{ }^{\circ} \mathrm{C}$, realizado en el zona mostrada en la Figura 7 , en él se observa la presencia de los elementos $\mathrm{Ca}$, $\mathrm{P}, \mathrm{O}, \mathrm{Fe}$, y Mo que constituyen dicho sólido, lo que nos indica la presencia de varias fases en el mismo debido al alto porcentaje atómico de los elementos presentes este puede corresponder a las fases anteriormente señaladas.

En la Figura 8, se presenta el resultado de los análisis de reducción a temperatura programada de los precursores catalíticos FeMo/Zn-hidroxiapatitas calcinados a $400^{\circ} \mathrm{C}$. En todos los perfiles fue posible 


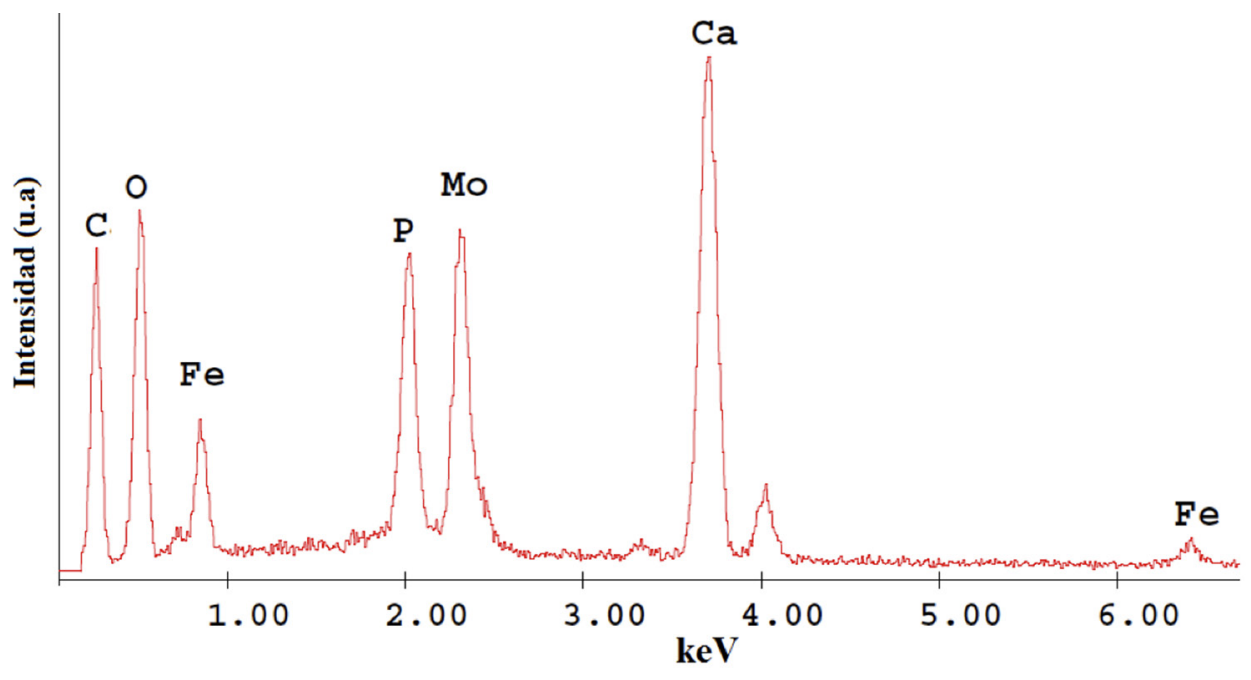

Figura 7. Espectro EDS de la muestra FeMo/Zn-hidroxiapatita ( $0 \% \mathrm{Zn}$ ) calcinada a $400{ }^{\circ} \mathrm{C}$.

observar dos señales perfectamente distinguibles: una señal "A" que aparece entre 500 y $700{ }^{\circ} \mathrm{C}$, y otra señal "B" que aparece entre 700 y $1100{ }^{\circ} \mathrm{C}$. Estas señales están relacionadas con las especies reducibles de molibdeno. Según Lee [15], el primer pico puede ser asignado a las especies octaédricas de Mo reducible, y el segundo pico, puede deberse a especies tetraédricas de Mo más difíciles de reducir. No se descarta, que este último pico esté acompañado por especies de fosfato las cuales pueden ser también reducibles a fosfito [13].

Para el precursor FeMo/alúmina, se destacan estos picos bien pronunciados: uno bien definido y con un alto consumo de $\mathrm{H}_{2}$ a $500{ }^{\circ} \mathrm{C}$ y uno ancho a $733^{\circ} \mathrm{C}$. Se observa que el pico correspondiente a las especies octaédricas (señal de baja temperatura) presentó mayor consumo de hidrógeno que aquel asignado a las especies tetraédricas (pico de alta temperatura). La señal "A" identificada para el precursor soportado en alúmina comercial se redujo a temperaturas menores que aquellos mostrados en la señal "B", los cuales se redujeron a temperaturas muy similares a los observados en FeMo/alúmina. La razón de que estas señales aparezcan a temperaturas menores en la alúmina, es debido a que las interacciones metalsoporte son más débiles que aquellas observadas en la hidroxiapatita. Para los precursores FeMo/Znhidroxiapatitas, la señal "A" se observó en el intervalo entre 570 y $700{ }^{\circ} \mathrm{C}$ y la señal "B" en el intervalo correspondiente entre 750 y $1100{ }^{\circ} \mathrm{C}$. A medida que aumenta el contenido de cinc, la temperatura de reducción de la señal "A" correspondiente al molibdeno octaédrico, responsable de la actividad catalítica en los catalizadores de HDS, es desplazada a temperaturas mayores sugiriendo que se hace más difícil de reducir.

En la Tabla 3 se muestran las medidas de las señales "A" y "B" de los reductogramas de los precursores catalíticos calcinados a $400{ }^{\circ} \mathrm{C}$. Se puede observar que el catalizador $\mathrm{FeMo/alúmina} \mathrm{presentó} \mathrm{la} \mathrm{relación}$ de las señales A/B más alta, seguido de los sólidos con 3, 0 y $6 \%$ en $\mathrm{Zn}$, que presentaron relaciones de señales A/B alrededor de 1. Por consiguiente, deberían

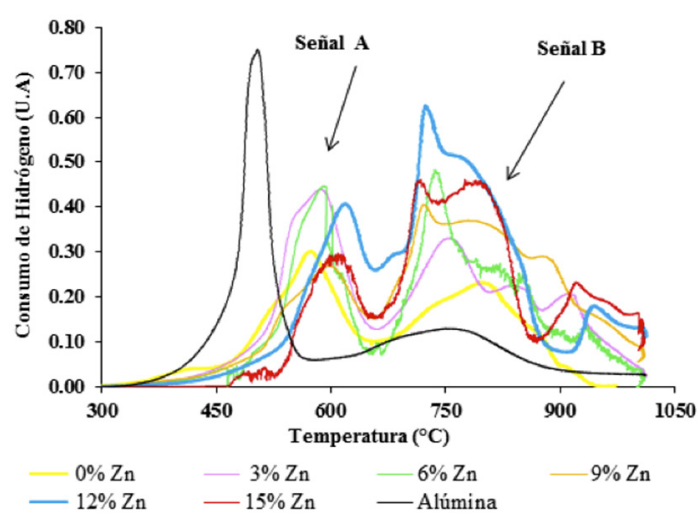

Figura 8. Reductogramas de las muestras $\mathrm{FeMo} /$ Zn-hidroxiapatitas calcinadas a $400{ }^{\circ} \mathrm{C}$. 
tener una mayor actividad en las reacciones de HDS, ya que poseen una mayor cantidad de Mo octaédrico en su estructura. Asimismo, las muestras con 9, 12 y $15 \%$ en $\mathrm{Zn}$ mostraron relaciones menores a uno y bastante parecidas entre sí, indicando que la cantidad de Mo tetraédrico estuvo en una mayor proporción. La relación de las señales A/B disminuye a medida que aumenta el contenido de cinc, lo que implica que la señal correspondiente a molibdeno tetraédrico, el cual es más difícil de reducir, se va incrementando.

Los sólidos con bajo contenido en Zn parecieran tener una mayor dispersión de la fase activa (Mo) producto de la presencia de iones $\mathrm{HPO}_{4}{ }^{2-}$ de la hidroxiapatita [14]. A medida que se incrementa el contenido de cinc se favorece la formación de sales de fosfato doble de calcio y cinc $\mathrm{Ca}_{19} \mathrm{Zn}_{2}\left(\mathrm{PO}_{4}\right)_{14}$, y la aparición de las fases scheelita $\mathrm{CaMoO}_{4}$, ferrimolibdito $\mathrm{Fe}_{2} \mathrm{Mo}_{3.9} \mathrm{O}_{14.7} 10.5 \mathrm{H}_{2} \mathrm{O}$ y molibdato férrico $\mathrm{Fe}_{2}\left(\mathrm{MoO}_{4}\right)_{3}$, poco reducibles, lo cual implica una baja concentración de la fase activa en conjunto con el efecto de "coreshell" que pudiese estar ejerciendo el fosfato de calcio y cinc. En este caso, el fosfato de calcio cubririría, en forma de una delgada capa a los sitios de Mo octaédrico impidiendo su reducción [15].

En la Figura 9, se presenta el resultado de los análisis de TPR de los precursores catalíticos calcinados a $800{ }^{\circ} \mathrm{C}$.

Para el precursor FeMo/alúmina se destacan tres picos a 438, 600 y $733{ }^{\circ} \mathrm{C}$. El primer pico ha sido asignado a especies poliméricas de óxido de molibdeno octaédrico fáciles de reducir a $438{ }^{\circ} \mathrm{C}$ y el último pico a $733{ }^{\circ} \mathrm{C}$, corresponde a especies aisladas de óxido de molibdeno tetraédrico más

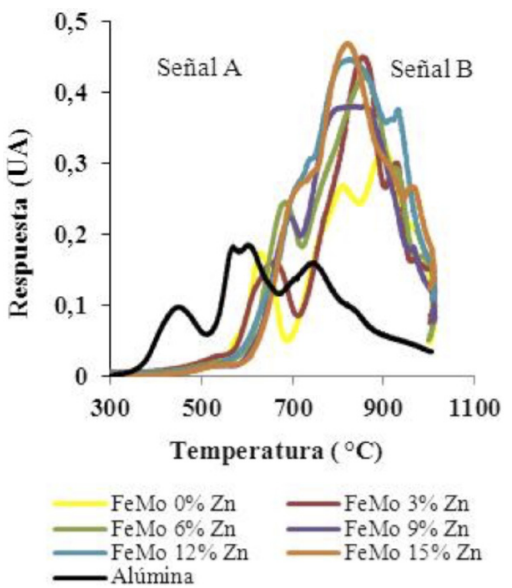

Figura 9. Reductogramas de las muestras $\mathrm{FeMo} /$ Zn-hidroxiapatita calcinadas a $800^{\circ} \mathrm{C}$.

difíciles de reducir [15]. La señal a $600{ }^{\circ} \mathrm{C}$ podría ser asignada a especies de hierro reducibles.

En lo que respecta a los reductogramas de los precursores catalíticos con 0 hasta $15 \%$ en $\mathrm{Zn}$, lucen complejos y no se observa una tendencia clara. En todos ellos se pueden observar, básicamente, un hombro entre 600 y $780{ }^{\circ} \mathrm{C}$, con excepción de los precursores con 12 y $15 \%$ en $\mathrm{Zn}$ donde se observa un pico en el intervalo de 800 y $850{ }^{\circ} \mathrm{C}$, y un hombro más pequeño entre 950 y $1000^{\circ} \mathrm{C}$. Con respecto al pico observado en el intervalo de 800 y $850^{\circ} \mathrm{C}$, éste se hizo más intenso y el consumo de hidrógeno se incrementó a medida que aumentó el contenido de cinc en la estructura. Se puede decir que este pico podría ser una combinación de muchas especies poco reducibles tales como: Mo tetraédrico, especies de Zn, fosfatos de calcio,

Tabla 3. Consumo de hidrógeno y temperatura de reducción de los picos relacionados con la señal "A" y "B" de las muestras FeMo/Zn-hidroxiapatitas calcinadas a $400{ }^{\circ} \mathrm{C}$.

\begin{tabular}{|l|c|c|c|c|c|}
\hline \multirow{2}{*}{ Muestras } & \multicolumn{2}{|c|}{ Señal A } & \multicolumn{2}{c|}{ Señal B } & \multirow{2}{*}{$\begin{array}{c}\text { Relación } \\
\text { Señal A/B }\end{array}$} \\
\cline { 2 - 5 } & $\begin{array}{c}\text { Consumo de } \mathbf{H}_{\mathbf{2}} \\
\text { u.a }\end{array}$ & $\begin{array}{c}\text { Temperatura } \\
\mathbf{\pm \mathbf { 1 } ^ { \mathbf { 0 } } \mathbf { C }}\end{array}$ & $\begin{array}{c}\text { Consumo de } \mathbf{H}_{\mathbf{2}} \\
\text { u.a }\end{array}$ & $\begin{array}{c}\text { Temperatura } \\
\mathbf{\mathbf { 1 }} \mathbf{1}^{\mathbf{0}} \mathbf{C}\end{array}$ & \\
\hline FeMo/Hap (0\% Zn) & 0,300 & 571 & 0,233 & 796 & 1,28 \\
\hline FeMo/Hap (3\% Zn) & 0,429 & 577 & 0,324 & 744 & 1,32 \\
\hline FeMo/Hap (6\% Zn) & 0,435 & 582 & 0,469 & 735 & 0,92 \\
\hline FeMo/Hap (9\% Zn) & 0,262 & 587 & 0,395 & 716 & 0,66 \\
\hline FeMo/Hap (12\% Zn) & 0,394 & 609 & 0,616 & 721 & 0,64 \\
\hline FeMo/Hap (15\% Zn) & 0,287 & 696 & 0,616 & 710 & 0,46 \\
\hline FeMo/Alúmina & 0,737 & 500 & 0,125 & 733 & 5,90 \\
\hline
\end{tabular}


ferrimolibdito $\mathrm{Fe}_{2} \mathrm{Mo}_{3.9} \mathrm{O}_{14.7} 10.5 \mathrm{H}_{2} \mathrm{O}$, molibdato férrico $\mathrm{Fe}_{2}\left(\mathrm{MoO}_{4}\right)_{3}$, y la scheelita $\mathrm{CaMoO}_{4}$ tal como lo confirma el difractograma de rayos $\mathrm{X}$ con la presencia de estas fases. El hombro pequeño en el intervalo 950 y $1000{ }^{\circ} \mathrm{C}$ puede ser evaluado como la reducción de las especies de fosfato de calcio y cinc $\mathrm{Ca}_{19} \mathrm{Zn}_{2}\left(\mathrm{PO}_{4}\right)_{14}$, que se reducen a altas temperaturas $[13,16]$.

En la Tabla 4 se muestran las señales "A" y "B" de los TPR de los precursores catalíticos calcinados a $800^{\circ} \mathrm{C}$. Se puede observar que los precursores con contenidos entre 0 y $9 \%$ de $\mathrm{Zn}$ mostraron relaciones de las señales A/B menores a uno y los precursores con 12 y $15 \%$ de $\mathrm{Zn}$ no presentaron relación de las señales $\mathrm{A} / \mathrm{B}$, indicando que la cantidad de Mo tetraédrico estuvo en una mayor proporción. La relación de las señales $\mathrm{A} / \mathrm{B}$ permanecen prácticamente constante a medida que aumenta el contenido de cinc, con excepción del precursor con $3 \%$ en cinc.

Al comparar la proporción de Mo octaédrico a Mo tetraédrico (relación de las señales A/B) se puede afirmar que la cantidad de Mo tetraédrico es muchísimo mayor que la existente de Mo octaédrico para todas las muestras analizadas. Por consiguiente, los catalizadores de esta serie, deberían tener poca actividad en la reacción de HDS de tiofeno. La calcinación del precursor catalítico a $800^{\circ} \mathrm{C}$ afectó notablemente la reducción de las especies activas de Mo haciéndolas más difíciles de reducir; y así la mayoría del Mo octaédrico reducible se transformó a especies de Mo poco reducibles como la scheelita u otras especies de Mo tetraédrico [6].

En la Figura 10 se muestra la actividad específica (mol de tiofeno convertido* $\mathrm{h}^{-1 * \mathrm{~g}^{-1}}$ ) obtenida una vez alcanzado el estado estacionario de los sistemas en estudio.

Para los catalizadores calcinados a $400{ }^{\circ} \mathrm{C}$, se observa que el catalizador de $0 \% \mathrm{Zn}$ posee la más alta actividad, seguido del catalizador de $6 \% \mathrm{Zn}$ y del catalizador soportado en alúmina; posteriormente siguen los catalizadores de $3 \%$, y $9 \%$ y, por último, los de $12 \%$ y $15 \%$ de cinc. Resultado que refleja el compromiso entre la superficie específica y la relación entre los sitios de Mo tetraédricos y octaédricos; así el catalizador con $0 \%$ en cinc, aunque tiene una relación de las señales de reducción $\mathrm{A} / \mathrm{B}$ menor, en comparación con el catalizador a $3 \%$ de $\mathrm{Zn}$, presenta una mayor actividad. Hecho que se resalta con el catalizador al $6 \%$ de $\mathrm{Zn}$, el cual, aunque presenta una relación A/B cercana a 1 , al tener la mayor superficie específica, se refleja en una mayor actividad.

Es posible que para este catalizador en particular $(6 \%$ en Zn) se presente una composición óptima de Zn que permite una mayor actividad de este catalizador, debido a que resultados similares fueron obtenidos para la serie $\mathrm{FeMo} / \mathrm{Zn}$ - hidroxiapatitas calcinadas a $800^{\circ} \mathrm{C}$. Luego, y a medida que se incrementa el contenido de Zn, se observa una disminución progresiva de la actividad catalítica. Este comportamiento podría estar relacionado con una mayor proporción de las fases ferrimolibdito $\mathrm{Fe}_{2} \mathrm{Mo}_{3.9} \mathrm{O}_{14.7} 10.5 \mathrm{H}_{2} \mathrm{O}$ y molibdato férrico $\mathrm{Fe}_{2}\left(\mathrm{MoO}_{4}\right)_{3}$, cuya presencia da lugar a una disminución de la concentración de la fase activa, afectando la actividad catalítica en la reacción de HDS de tiofeno.

Posiblemente cuando el sulfuro de molibdeno es promovido con hierro, un efecto sinergético es obtenido, debido a la formación de nuevas fases,

Tabla 4. Consumo de hidrógeno y temperatura de reducción de los picos relacionados con la señal "A" y "B" de las muestras FeMo/Zn-hidroxiapatitas calcinadas a $800{ }^{\circ} \mathrm{C}$.

\begin{tabular}{|c|c|c|c|c|c|}
\hline \multirow[b]{2}{*}{ Muestras } & \multicolumn{2}{|c|}{ Señal A } & \multicolumn{2}{|c|}{ Señal B } & \multirow{2}{*}{$\begin{array}{c}\text { Relación } \\
\text { Señal A/B }\end{array}$} \\
\hline & $\begin{array}{c}\text { Consumo de } \mathrm{H}_{2} \\
\text { u.a }\end{array}$ & $\begin{array}{c}\text { Temperatura } \\
\pm 1^{\circ} \mathrm{C} \\
\end{array}$ & $\begin{array}{c}\text { Consumo de } \mathrm{H}_{2} \\
\text { u.a }\end{array}$ & $\begin{array}{c}\text { Temperatura } \\
\pm 1^{\circ} \mathrm{C} \\
\end{array}$ & \\
\hline $\mathrm{FeMo} / \mathrm{Hap}(0 \% \mathrm{Zn})$ & 0,173 & 632 & 0,269 & 809 & 0,64 \\
\hline $\mathrm{FeMo} / \mathrm{Hap}{ }_{(3 \% \mathrm{Zn})}$ & 0,154 & 643 & 0,438 & 845 & 0,35 \\
\hline $\mathrm{FeMo} / \mathrm{Hap}(6 \% \mathrm{Zn})$ & 0,245 & 678 & 0,420 & 851 & 0,58 \\
\hline FeMo/Hap $(9 \%$ Zn) & 0,221 & 688 & 0,380 & 850 & 0,58 \\
\hline FeMo/Hap $(12 \% \mathrm{Zn})$ & 0,00 & No aplica & 0,430 & 801 & 0,00 \\
\hline FeMo/Hap $(15 \%$ Zn $)$ & 0,00 & No aplica & 0,451 & 806 & 0,00 \\
\hline FeMo/Alúmina & 0,095 & 438 & 0,157 & 733 & 0,60 \\
\hline
\end{tabular}




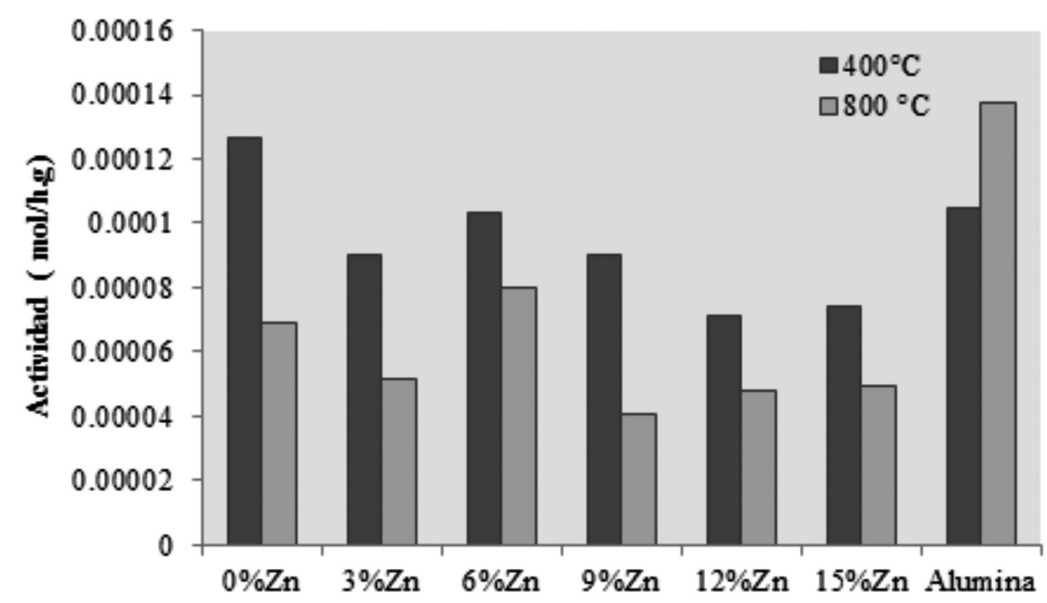

Figura 10. Actividad específica en la reacción HDS de tiofeno de los catalizadores FeMo/Zn-hidroxiapatitas calcinados a 400 y $800{ }^{\circ} \mathrm{C}$ en función del contenido de $\mathrm{Zn}$.

lo que demostraría el efecto promotor efectivo que ejerce el hierro sobre el molibdeno, a pesar de las bajas actividades obtenidas.

Para los catalizadores calcinados a $800{ }^{\circ} \mathrm{C}$, se aprecia que el catalizador soportado en alúmina es el que presenta la mayor actividad, luego se presenta el siguiente orden de actividad: $6 \%>0 \%>3 \%>12 \% » 15 \%>9 \%$. En esta serie de catalizadores se conjugan dos factores: una superficie específica muy pequeña, y todas las relaciones A/B menores a 1; es por ello, que las actividades son bajas y prácticamente constantes.

A excepción del catalizador al $6 \% \mathrm{Zn}$, que por las razones anteriormente señaladas, no sigue la tendencia presentada por esta serie.

En general, las actividades para las dos series de catalizadores estudiadas son bajas, como se había mencionado anteriormente. Para que los sólidos en estudio presenten actividad en la reacción de hidrodesulfuración de tiofeno, es necesaria la presencia de especies de molibdeno octaédrico y un soporte adecuado para la dispersión de la fase activa. Por otra parte, la superficie específica disponible también puede afectar la actividad catalítica [14].

En la Figura 11 se muestra la actividad intrínseca (mol de tiofeno convertido $* \mathrm{~h}^{-1 *} \mathrm{~m}^{-2}$ ) obtenida una vez alcanzado el estado estacionario de los sistemas en estudio.
Al observar la tendencia de los catalizadores calcinados a $400{ }^{\circ} \mathrm{C}$, se aprecia que este valor tiende a hacerse constante, lo que indica que prácticamente todos los sólidos tienen igual rendimiento por superficie específica expuesta al tiofeno para obtener los productos, existiendo un efecto de la superficie específica, tendencia no observada cuando se expresa en función de la masa de catalizador.

En cuanto a los catalizadores calcinados a $800^{\circ} \mathrm{C}$, la tendencia de los mismos es muy parecida a la actividad mostrada en función de la masa de catalizador, a excepción del catalizador al 12\% de Zn, el cual presentó la mayor superficie específica. Se observa que los catalizadores con contenido entre 0 y $15 \%$ de $\mathrm{Zn}$ con baja superficie específica $\left(4 \mathrm{~m}^{2 *} \mathrm{~g}^{-1}\right)$, mostraron una actividad intrínseca superior a la del catalizador comercial, lo cual implica que su actividad catalítica por superficie de contacto $\left(\mathrm{mmol}^{*} \mathrm{~g}^{-1 *} \mathrm{~m}^{-2}\right)$ es superior, lo cual pudiera deberse a que hay mayor cantidad de fase activa (FeMoS), por unidad de superficie de contacto.

\section{CONCLUSIONES}

La temperatura de calcinación de hidroxiapatitas con contenido variable de cinc afecta las medidas de área específica y el tamaño de los cristalitos. A medida que aumenta la temperatura de calcinación $\left(800^{\circ} \mathrm{C}\right)$, las medidas de superficie BET son menores que aquellas muestras calcinadas a $400^{\circ} \mathrm{C}$; sin embargo, el tamaño de cristal aumenta a medida que aumenta la temperatura de calcinación. 


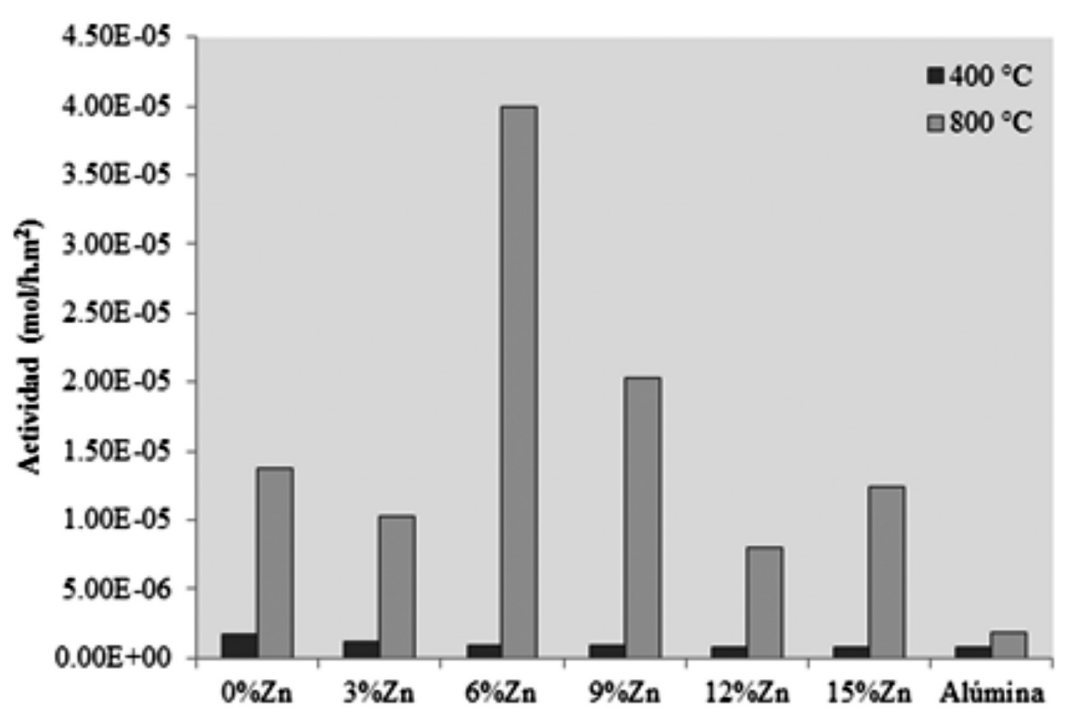

Figura 11. Actividad intrínseca en la reacción HDS de tiofeno de los catalizadores FeMo/Zn-hidroxiapatitas calcinados a 400 y $800{ }^{\circ} \mathrm{C}$ en función del contenido de Zn.

Estas Zn-hidroxiapatitas impregnadas con sales de Mo y Fe y con posterior calcinación a 400 y a $800^{\circ} \mathrm{C}$, dio lugar a la presencia de las siguientes fases cristalinas: $\mathrm{Fe}_{2} \mathrm{Mo}_{3.9} \mathrm{O}_{14.7} 10.5 \mathrm{H}_{2} \mathrm{O}, \mathrm{Fe}_{2}\left(\mathrm{MoO}_{4}\right)_{3}$, $\mathrm{Ca}_{19} \mathrm{Zn}_{2}\left(\mathrm{PO}_{4}\right)_{14}$ y $\mathrm{CaMoO}_{4}$.

La calcinación de estas hidroxiapatitas afecta la proporción de especies de Mo octaédrica y Mo tetraédrica, siendo la proporción de especies de Mo octaédrica superior cuando la temperatura de calcinación es menor $\left(400^{\circ} \mathrm{C}\right)$.

A mayor contenido de $\mathrm{Zn}$ dentro de la estructura de la hidroxiapatita, la tendencia es aumentar la proporción de especies de Mo octaédrica.

La actividad catalítica de la reacción de HDS de tiofeno fue afectada por la temperatura de calcinación, los sólidos calcinados a $800{ }^{\circ} \mathrm{C}$ fueron menos activos que aquellos calcinados a $400^{\circ} \mathrm{C}$, debido a las menores áreas específicas y a la presencia de un mayor contenido de especies de Mo tetraédrica.

\section{AGRADECIMIENTOS}

Los autores agradecemos al Fonacit (PAEFONACIT $\mathrm{N}^{\circ}$ 2011000797) por el financiamiento otorgado para llevar a cabo este proyecto.

\section{REFERENCIAS}

[1] C. Marcilly. "Evolution of refining and petrochemicals. What is the place of zeolites". Studies in Surface Science and Catalysis, Elsevier. Vol. 135, pp. 37-60. Date of visit: June, 2011. URL: ISSN 0167-2991. https:// doi.org/10.1016/S0167-2991(01)81185-X

[2] G. Alves, R. García y R. Cid. "Arcillas pilareadas II. Hidrodesulfuración de tiofeno sobre catalizadores $\mathrm{Ni}$, W soportados sobre arcillas pilareadas". Boletín de la Sociedad Chilena de Química. Vol. 44 Nº 3, pp. 37-344. Fecha de consulta: Septiembre, 1999. URL: ISSN 0366-1644. https://dx.doi.org/10.4067/ S0366-16441999000300010

[3] T. Klimova, M. Calderón and J. Ramirez. "Ni and Mo interaction with Al-containing MCM-41 support and its effect on the catalytic behavior in DBT hydrodesulfurization". Applied Catalysis A General. Vol. 240 No 1-2, pp. 29-40. Date of visit: February, 2003. URL: ISSN 0926-860X. https://doi.org/10.1016/ S0926-860X(02)00417-9

[4] F. Gonzalez-Jimenez, H. Constant, R. Iraldi, E. Jaimes and M. Rosa-Brussin. "Mössbauer study of hydrodemetallization clay catalysts of heavy oils: Role of pyrrhotites". Hyperfine 
Interactions. Vol. $28 \mathrm{~N}^{\circ}$ 1, pp. 927-930. Date of visit: February, 1986. URL: ISSN 03043843. https://doi.org/10.1007/BF02061596 L. Lippolis y M.A Luis. "Reacciones de HYD del 2MN, HDS de DBT y competitiva en condiciones de baja severidad empleando sulfuros másicos mixtos de $\mathrm{Fe}-\mathrm{Nb}$ no frescos". Revista Ingeniería UC. Vol.17 $\mathrm{N}^{\circ}$ 1, pp. 61-72. Fecha de visita: Abril, 2010. URL: ISSN 1316-6832. http://www. redalyc.org/articulo.oa?id $=70721861008$

[6] C.F. Linares, E. Figueredo, D. Peraza G. González y F. Ocanto. "Efecto de la temperatura de calcinación en catalizadores NiMo/Zn-Hidroxiapatitas en la reacción de hidrodesulfuración de tiofeno". Revista Latinoamericana de Metalurgia y Materiales. Vol. $34 \mathrm{~N}^{\mathrm{o}}$ 1, pp. 66-75. Fecha de consulta: Enero, 2014. URL: ISSN 255-69521. www. scielo.org.ve/pdf/rlmm/v34n1/art07.pdf

[7] B. García, G. González, F. Ocanto y C.F Linares. "Influencia de la temperatura de calcinación en la síntesis de potenciales catalizadores de hidrotratamiento a base de CoMo/Zn-hidroxiapatitas". Acta Microscópica. Vol. $20 \mathrm{~N}^{\mathrm{o}}$ 2, pp. 147-156. Fecha de consulta: Noviembre, 2011. URL: ISSN: 0798-4545. https://www. researchgate.net/publication/281570011 INFLUENCIA_DE_LA_TEMPERATURA DE_CALCINACCION_EN_LA_SINTESIS DE_POTENCIALES_CATALIZADORES DE_HIDROTRATAMIENTO_A_BASE_ DE_CoMoZn_HIDROXIAPATITAS

[8] F. Miyaji, Y. Kono and Y. Suyama. "Formation and structure of zinc-substituted calcium hydroxyapatite". Materials Research Bulletin. Vol. 40 No $^{\circ}$, pp. 209-220. Date of visit: February, 2005. URL: ISSN 0025-5408, https://doi.org/10.1016/j. materresbull.2004.10.020

[9] R. LeGeros M. Taheri G. Quirolgico and J.P. LeGeros. "Formation and stability of apatites: effects of some cationic substituents". $2^{\text {nd }}$ International Congress on Phosphorus Compounds. Estados Unidos de América. 1980.

[10] U. Spadavecchia y G. González. "Obtención de hidroxiapatita nanométrica para aplicaciones médicas". Revista Facultad de Ingeniería Universidad Central de Venezuela. Vol. 22
$\mathrm{N}^{\circ}$ 4, pp. 37-44. 2007. ISSN 0798-4065. http:// www.scielo.org.ve/scielo.php?script=sci_ arttext\&pid=S0798-40652007000400004\&ln $\mathrm{g}=\mathrm{es} \& \mathrm{nrm}=\mathrm{iso} \&$ tlng=es

[11] Y. Saih, M. Ait Chaoui, A. Ezzamarty and M. Lakhdar. "Based CoMo sulphided catalysts supported on hydroxyapatite: Dimethyldisulphide (DMDS) hydrogenolysis". Catalysis Communications. Vol. $2 \mathrm{~N}^{\mathrm{o}}$ 2, pp. 81-87. Date of visit: May, 2001. URL: ISSN 1566-7367. http://dx.doi. org/10.1016/S1566-7367(01)00007-3

[12] I. Cacciotti, A. Bianco, M Lombardi and L. Montanaro. "Mg-substituted hydroxyapatite nanopowders: Synthesis, thermal stability and sintering behavior". Journal of the European Ceramic Society. Vol. $29 \mathrm{~N}^{\mathrm{o}} 14$, pp. 2869-2978. Date of visit: November, 2009. URL: ISSN 0955-219. https://doi. org/10.1016/j.jeurceramsoc.2009.04.038

[13] J. Guerra, J. Brito, G. González, R. Villalba, M. Pagano y J. Sánchez. (2008). “Uso catalítico de hidroxiapatitas sustituidas con vanadato y molibdato en HDS de tiofeno". XXI Simposium Iberoamericano de Catálisis. España, Málaga. 2008.

[14] N. Elazarifi, M. Aït Chaoui, A. El Ouassouli, A. Ezzamarty, A. Travert, J. Leglise, L. de Ménorval and C. Moreau. "Hydroprocessing of dibenzothiophene, 1-methylnaphthalene and quinoline over sulfided NiMo-hydroxyapatite-supported catalysts". Catalysis Today. Vol. 98 No 1-2, pp. 161-170. Date of visit: November, 2004. URL: ISSN 0920-5861, http://dx.doi. org/10.1016/j.cattod.2004.07.030

[15] J.J. Lee, H. Kim and S.H. Moon. "Preparation of highly loaded, dispersed $\mathrm{MoS}_{2} / \mathrm{Al}_{2} \mathrm{O}_{3}$ catalysts for the deep hydrodesulfurization of dibenzothiophenes". Applied Catalysis B: Environmental. Vol. $41 \mathrm{~N}^{\circ}$ 1-2, pp. 171180. Date of visit: March, 2003. URL: ISSN 0926-3373, https://doi.org/10.1016/ S0926-3373(02)00209-6

[16] J. Medema, C. van Stam, V.H.J. de Beer, A.J.A. Konings and D.C. Koningsberger. "Raman spectroscopic study of CoMoy$\mathrm{Al}_{2} \mathrm{O}_{3}$ catalysts, Journal of Catalysis". Vol. $53 \mathrm{~N}^{\mathrm{o}} 3$, pp. 386-400. Date of visit: July, 1978. URL: ISSN 0021-9517, https:// doi.org/10.1016/0021-9517(78)90110-0 